\title{
CATASTROPHIC ANTIPHOSPHOLIPID SYNDROME WITH HEMORRHAGIC ALVEOLITIS
}

Andrea Worm Furtado ${ }^{1, \star}$, Afonso Guilherme Schmidt ${ }^{1}$, Larissa Vargas Cruz ${ }^{1}$, André Lucas Ribeiro ${ }^{1}$, Augusto Emílio Hinterholz ${ }^{1}$, Larissa Martinelli Dullius ${ }^{1}$, Vanessa Hax ${ }^{1}$, Ilka Benedet Lineburger ${ }^{1}$, Ricardo Machado Xavier ${ }^{1}$

1.Universidade Federal do Rio Grande do Sul, Porto Alegre (RS), Brazil.

*Corresponding author: andreawfurtado@yahoo.com.br

\section{BACKGROUND}

Catastrophic antiphospholipid (CAPS) is a rare complication of antiphospholipid syndrome (APS) and is characterized by the rapid development of multiple organ or system thrombosis. We report a very rare case of CAPS complicated with hemorrhagic alveolitis and myocarditis.

\section{CASE REPORT}

A 59-year-old female patient with CREST syndrome, presenting thickening of the skin, Raynaud's phenomenon, nailfold capillaroscopy with scleroderma pattern, telangiectasias and anti-centromere antibody, and APS with a history of two episodes of lower extremity deep vein thrombosis, two ischemic strokes and a positive lupus anticoagulant, anticardiolipin IgM (> 225 UI) and IgG (> $280 \mathrm{UI}$ ), attended the emergency department with a one-day history of pleuritic chest pain and sudden bilateral visual loss. She was taking apixaban instead of warfarin by her own decision after a subdural hematoma 7 years before. Ophthalmological evaluation showed macular ischemic areas and embolic occlusion of arterial branches in both eyes and cardiac nuclear magnetic resonance showed myocarditis. Despite methylprednisolone $1 \mathrm{mg} / \mathrm{kg} / \mathrm{day}$ and anticoagulation with heparin, the patient evolved with thrombocytopenia $\left(60,000 / \mathrm{mm}^{3}\right)$, new areas of retinal thrombosis and loss of strength and sensitivity in the 4 th and 5 th right fingers, with a diagnosis of ulnar nerve mononeuritis. After three days of IV methylprednisolone $1 \mathrm{~g}$, she developed dyspnea, hypoxemia, and anemia (7 mg/dL), lymphopenia, renal disfunction with proteinuria and dysmorphic hematuria, worsening thrombocytopenia $\left(30,000 / \mathrm{mm}^{3}\right)$ and low complement. Bronchoalveolar lavage confirmed hemorrhagic alveolitis and excluded infectious causes. Anticoagulation was interrupted. Abdomen and chest CT were performed with evidence of bilateral ground glass opacities, pulmonary thromboembolism, and thrombosis in the iliac and femoral arteries. The patient received plasma exchange sessions interspersed with another IV steroid pulse therapy for five days. Clinical and laboratory findings improved. Heparin anticoagulation was restarted and subsequently switched to oral warfarin. Due to the possibility of catastrophic APS associated with systemic lupus erythematosus, she received the first infusion of cyclophosphamide and was discharged from hospital with prednisone $1 \mathrm{mg} / \mathrm{kg} /$ day. Two months later, the patient returned to the emergency room with dyspnea and died after cardiorespiratory arrest during a central venous access procedure.

\section{CONCLUSION}

Catastrophic antiphospholipid is a rheumatologic emergency, whose optimal treatment is still unknown. Despite anticoagulants, steroids, intravenous immunoglobulin, and plasma exchange, the mortality rate is still nearly $50 \%$. We present a rare case of CAPS with complicated hemorrhagic alveolitis. Our case demonstrates the various clinical features and therapeutic difficulties encountered by rheumatologists while diagnosing and treating this life-threatening condition.

\section{KEYWORDS}

Catastrophic, Antiphospholipid antibody, Thrombosis. 